\title{
The Evolution and Recent Progress in the Field of Synthetic Immunology
}

\author{
Amaan Rather ${ }^{1}$ and Pavithran Ravindran ${ }^{\#}$ \\ ${ }^{1}$ Caesar Rodney High School, Camden, DE, USA \\ \#Advisor
}

ABSTRACT

Synthetic immunology is a field in which researchers design constructs that will help immune cells battle pathogens, most commonly cancer cells. This is particularly crucial for human health due to the considerable number of ways that invaders (to the body) possess to minimize the effectiveness of the immune system. Frequently, these changes take place in the form of developing more advanced synthetic receptors for better recognition of pathogens so that Tcells can execute more precise functions in the body. Other changes are also made to give researchers more control over the advancements that have been inserted into the body, heightening the level of safety for the patients who receive them. Considering the newfound research that has been conducted, this paper focuses on the significance of upgrading various parts of the immune system in terms of the way that they can help protect the body. It also highlights the extensive potential this field has in the future considering the adaptability and functionality of the current, newlydesigned systems in place.

\section{Introduction}

The immune system is essential for the survival of all humans - its intricate construction allows for our bodies to initiate complex attacks to protect us from foreign invaders. Our immune system consists of a vast quantity of organs and cells that prevent us from getting sick. Some examples of organs that help to protect us include tonsils, organs located near the rear of the throat that prevent germs from entering through the nose or mouth, bone marrow, tissue found inside the bones that produce immune cells, and lymph nodes, small pieces of tissue that function as filters and are found along the lymphatic vessels (IQWiG, 2006). These organs are made up of a wide variety of immune cells, and researchers have begun to engineer multiple combinations of these cell types in order to prevent breakdowns in our body's defenses. One of the most significant types are lymphocytes, a specialized group of white blood cells that are found throughout the body. The most important types of lymphocytes are natural killer cells (important for quick responses or "natural killing" as they do not need to be primed by the presence of a foreign body), B Cells (which predominantly produce antibodies), and most importantly T-Cells (most of which most help kill tumor cells and help control immune responses by activating other cells of the immune system). Several other different immune cells have also been engineered; examples include regulatory T- Cells (deal with immunosuppression), macrophages (deal with vaccination and remodeling the microenvironment), and neutrophiles (deal with phagocytosis, the process of engulfing other cells or particles in the body) (Roybal and Lim, 2017). The study of synthetic immunology has given humans the ability to create complex synthetic structures to aid immune cells (mainly the cytotoxic, or "killer" T-Cells) in their goal of safety from outside threats (Roybal et al, 2016).

The "synthetic" improvements to the immune system can occur in many different forms: improving the receptor designs of the T-Cell (whose primary function is killing infected host cells), heightening the specificity of antigens (molecules on the exterior of infectious agents that allow the immune system to target them in defensive procedures while preventing autoimmune reactions where the body attacks itself) (Choe, Williams, and Lim, 2020), 
editing T-Cells to be more practical/safe for use in patients who could potentially benefit from them (Mo et al., 2020; Wu et al., 2015), and other changes to increase the reliability of other artificially created additions that are inserted into the body. The developments in this field are essential to human health and safety around the world and provide desperately needed treatments to several devastating ailments. This is because eliminating foreign bodies such as tumors is an extremely complex process in which many steps have to occur successfully and this is difficult with the many issues that tumors can generate (which will be discussed later on). Synthetic improvements boost the capabilities of the immune system back to its normal level, and in many cases can even give it abilities to perform functions that it would not normally be able to carry out. Cancers, along with other afflictions including viral/bacterial infections, all have the potential to be remedied via synthetic immunology and it is important that researchers continue to tap into the full potential of what this field has to offer. However, currently most experiments and advancements in synthetic immunology have been centered on cancer therapy, and that will be the focus of the paper. More recent advances in the field have already had enormous impacts on the ways patients are treated for various diseases and these have drastically changed the way we view immunology moving forward.

\section{Methodology}

Existing literature was initially chosen based on finding the fundamental papers in the field. These were selected through the utilization of key search words including "synthetic immunology", "engineered T-Cells", and "CARs". Careful consideration was used to identify what papers demonstrated the initial advancements of the field. Building upon this, additional papers were obtained utilizing more complex search terminology and searching major authors in the field (i.e. Roybal) for more recent/advanced research. In all, websites were scoured for various types of papers that covered concepts thought to be the most crucial in the field. The major websites accessed included Cell, PubMed, and ScienceDirect.

\section{Receptor Design- CAR's (Chimeric Antigen Receptors)}

The natural, inbuilt ability of the immune system to protect humans is undeniable; however, there are several changes that scientists have made specifically to immune cell receptors to improve the body's response to foreign bodies, namely solid tumors/cancers. Researchers have modified T-Cells to contain Chimeric Antigen Receptors (CAR's) which contain an extracellular antigen-binding domain on the cell surface (Choe, Williams, and Lim, 2020; Kuwana et al, 1987) (Figure 1A). This domain recognizes the cancer antigen while an intracellular signaling domain and costimulatory domain lead to T-Cell activation. This addition helps the T-Cell focus its cytotoxic power towards the tumor cells. Overall, CAR's allow T-Cells to more specifically attack a single cancer in order to eliminate it from the bloodstream.

However, even these receptors face serious issues that prevent them from successfully eliminating cancer cells. The lack of cancer-specific antigens to target lead to the possibility that CAR T-Cells may attack normal, healthy body tissue (Roybal and Lim, 2017). This situation arises because an antigen can be found in many different forms, including proteins, peptides, and polysaccharides. Many tumor cells and normal cells share antigens and this leaves the latter susceptible to being killed by the CAR-Cell (currently the main FDA approved T-Cell can only recognize the CD19 antigen, further restricting the practical applications of the system). Another complication is the creation of an immunosuppressive tumor microenvironment (TME). This refers to the ability of tumors to suppress the immune system's response using cell death ligands (signals), suppressive cytokines (proteins used in cell signaling), and physical barriers. Researchers have also experienced difficulty in ensuring the safety of all patients who receive synthetic CAR T-Cells due to the lack of accessibility over these cells in case any changes need to be made to their design or if they need to be removed. Therefore, it can be deciphered that recognition precision, the ability to overcome suppressive TME's, and user-control are all crucial features that fully functional CAR's must have. 
A

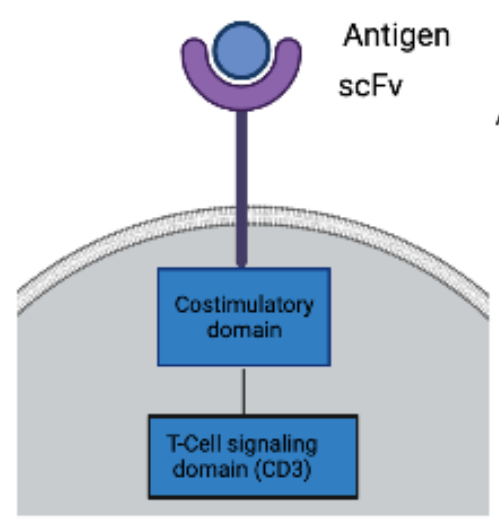

CAR T-Cell Structure

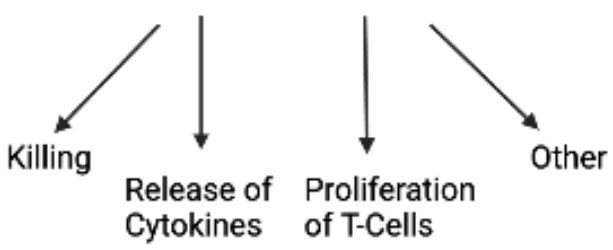

B

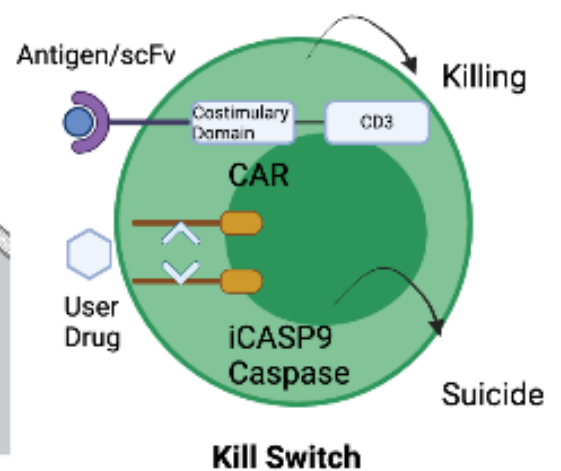

C

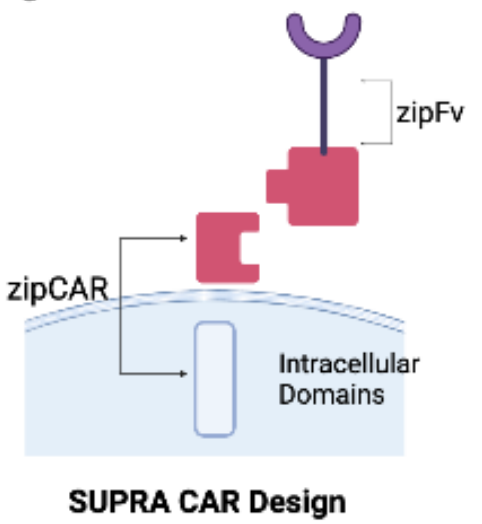

Figure 1: (A) The CAR has an extracellular binding domain in the form of a single-chain variable fragment. This is fused to an inside that contains a costimulatory domain with the normal T-Cell signaling domain (B) This demonstrates an example of using a split caspase 9 (iCASP9) with a small heterodimerizing drug to act as a "kill switch". This provides a layer of security in case the engineered cells need to be shut down (C) Not being able to fine tune T-Cell activation, enhance tumor precision, and independently control signaling pathways and cell types are some of the issues that standard CAR-T Cells face. The advanced twopart SUPRA CAR system contains a universal receptor in zipCAR and a scFv tumor-targeter in the zipFv. Both parts contain a leucine zipper, connected to the intracellular domains and $\mathrm{scFv}$ respectively. When activated, the scFv attaches to the antigen and the leucine zipper binds and activates the zipCAR. Created using BioRender.

One main method used to remedy these issues is combinatorial antigen recognition - programming CAR TCells to recognize multiple antigens at a time for more specific targeting (more on this in the antigen section). To overcome the immunosuppressive signals that many cancers send within the body, researchers have also used gene editing to reduce the inhibitory signaling capabilities of T-Cells (which reduce the cell's immune response) and engineered technology such as dominant-negative receptors that act as "sinks" that collect these signals and dispose of them (Rupp et al., 2017). An example of this can be seen with the TGF- $\beta$ (Transforming growth factor beta) receptor, which has shown the ability to overcome suppression initiated by TGF- $\beta$ (cytokine released by tumors that initiates apoptosis, or cell death) in various trial models. Other receptors also have the ability to rewire the body's response to inhibitory inputs allowing it to generate a stimulatory response (Yamamoto et al, 2019). Researchers have also been hesitant in altering the genetic profile of CAR T-Cells to induce a proliferate state long term because, as mentioned previously, there are a lack of ways to control these engineered cells. However, researchers have now developed "kill switches" in CAR T-Cells in case there arises a time in which they need to be shut down completely (Yu et al, 2019) (Figure 1B). This was accomplished by binding human caspases (enzymes important in programmed cell death) to binding proteins. This two-part system then only activates in presence of another small-molecule which can be inserted into the body via specific drugs. Other systems have also been developed to ensure specificity instead of killing the engineered cells (will be discussed later). The development of CAR T-Cells is extremely complex but their uses and payoff justify the risk of their implementation.

Further advancements have been made to CAR T-Cells that have broadened the applicability of their use in patients. Examples of this include a new "SUPRA CAR" system that involves installing special CAR's in T-Cells that 
have multiple functions (Cho, Collins, and Wong, 2018) (Figure 1C). The SUPRA CAR system is unique in the way that it is engineered - it contains multiple receptors and molecules that function as "tunable variables" in order to control the activities performed by the T-Cell. This expands the capabilities of CAR by implementing several upgrades to the receptor, including the ability to sense multiple combinations of antigens as opposed to just one, to control the activation strength of T-Cells, to switch the cells that the T-Cells are targeting, to independently control the roles of various T-Cell subsets, and more. Researchers have created this by abandoning the rigid design of CAR's. The original CAR possessed one single-chain variable fragment $(\mathrm{scFv})$ for recognition and one intracellular domain which prompts one particular response. On the flip side, the receptors now consist of a zipCAR (intracellular domain and a leucine zipper) and a "zipFv" which also has a leucine zipper and is connected to a scFV. When fused together the receptor is activated and depending on the type of zipFv the receptor can now target multiple different antigens. SUPRA CAR is special because researchers have several variables that they can control to edit the function of the system; these include affinity between the zipper pairs, the affinity between the antigen and scFV, the concentration of zipFv, and the expression level of zipCAR. These changes can all control how T-Cells are activated and how they act. Adaptations to this system help expand the flexibility, specificity, and sovereignty over CAR's and their impact on the body. This system can also control immune cells other than T-Cells. As a result, it presents an enormous amount of flexibility and allows for cancer treatment to be safer and more precise.

Additionally, researchers have developed a convertibleCAR T-Cell system to treat various diseases (even outside of cancer) like latent HIV (Herzig et al., 2019). Latent HIV Cells persist over time despite antiretroviral therapy (the standard HIV treatment regimen) and they remain hidden to the immune system as they do not display viral proteins. A solution to this problem is imperative, as doctors want to ensure "post-treatment control" in the patient so that the HIV won't be able to infect any other cells. In the body, there are several antibodies known as bNabs (broadly neutralizing antibodies) that can recognize HIV infected cells and help neutralize them, but they need to work in combination with the immune system in some way to be effective. Thus, researchers took the NKG2D receptor, which is present naturally in lymphocytes (and normally acts as a security system against malignant cells), and fused it with these antibodies to create a molecule known as a MicAbody. These MicAbody's are then engaged, or activated, by ligands that are highly present on cells that have been stressed by viral infection - this is known as the convertibleCAR T-Cell platform. The system was shown to be extremely effective in a mouse lymphoma model; only HIV infected cells were targeted and killed and this only happened when the HIV-specific MicAbody was present. This clearly demonstrated the control researchers have over the system. There are many different ways CAR systems could potentially be developed in the future - for example, if the CAR T-Cell could simply sense the abnormal growth and size of the tumor (bypassing the need for an antigen).

\section{Receptor Design- synNotch}

Another class of newly developed receptors that play a prominent role in the field of synthetic immunology are synthetic notch receptors (synNotch receptors) (Figure 2A). These are very similar to CAR's since they sense antigens and various other environmental cues in order to initiate various response programs. When they sense the antigen an extracellular transcription factor enters the cell to activate transcription, or gene expression of what the receptor was programmed to perform. They have more general applications and can even be used to control CAR's and normal TCell receptors (Roybal and Lim, 2017). This is because the sensing of an antigen is actually activating a transcriptional program, which is user defined and thus grants vast possibilities for the control of cells. As such, one of key advantages to using the synNotch system is its versatility and its ability to deliver a wide variety of therapeutic activities. One specific use of the system that could be intriguing would be to engineer the cell to locally deliver growth or regeneration factors in response to an antigen expressing that injury has occurred to a group of cells. 
A

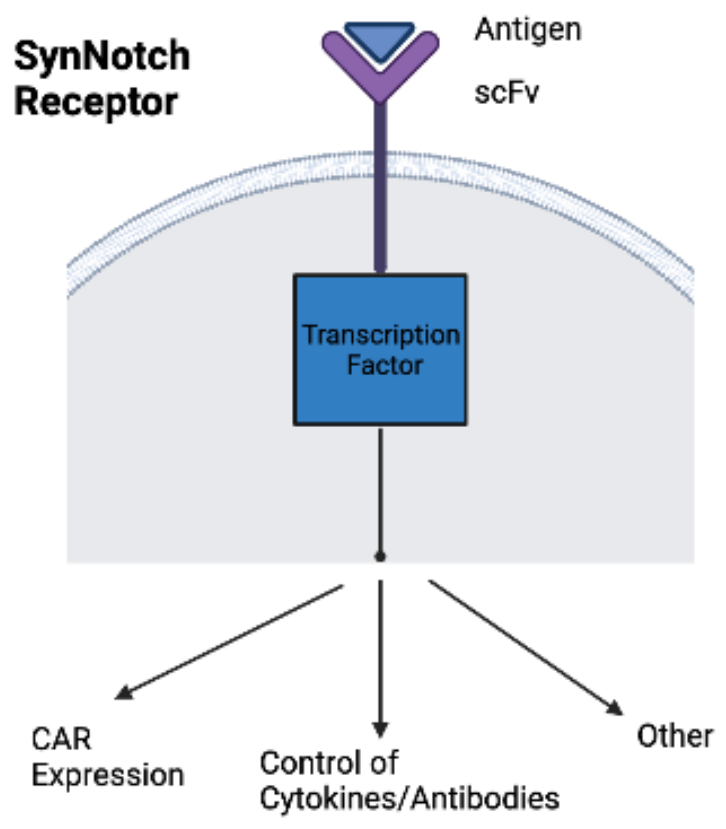

B
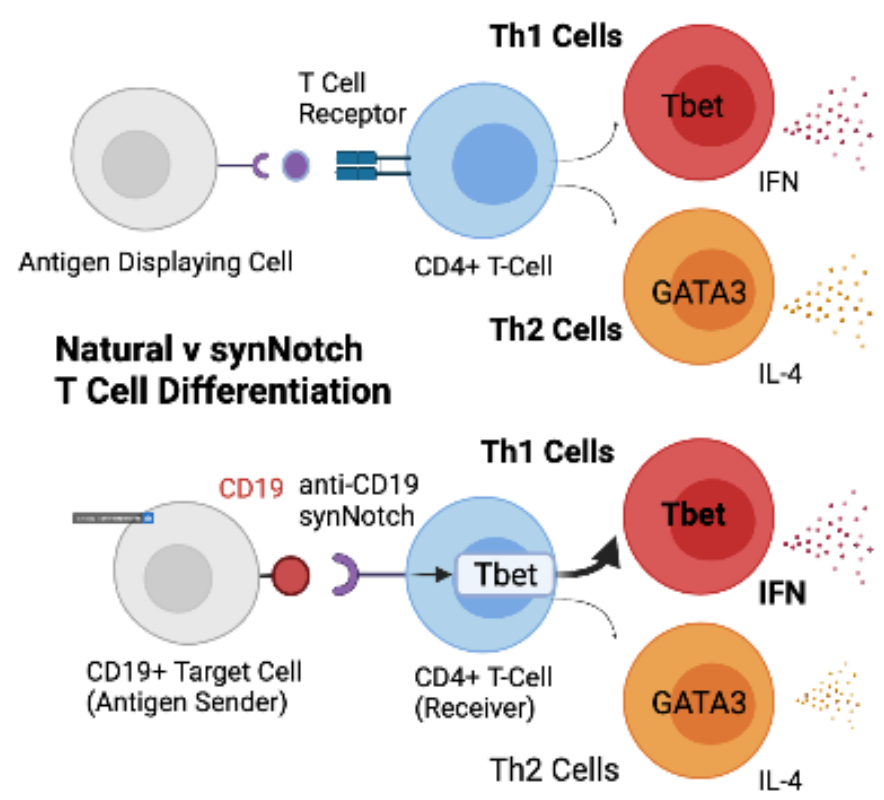

Figure 2: (A) The synNotch receptor contains an extracellular binding domain for the antigen/ligand in the form of a singlechain variable fragment. This then activates a transcription factor which acts as a signaling domain and can be programmed to activate a variety of tasks, as shown. They contain a structure very similar to the CAR receptors shown earlier (B) In many cases, T-Cell differentiation is skewed in a direction that makes them ineffective in fighting cancers. Here researchers used a synNotch that sensed the CD-19 antigen by increasing levels of Tbet. In turn, this produced more Th1 cells. This was intended as these cells are better equipped to clear tumors (while Th2 Cells focus on antibody response). Created using BioRender.

Currently, synNotch receptors can be used to deliver cytokines, antibodies, or even small molecules to various areas of the body in response to the antigens they sense (Roybal et al, 2016). A practical example of this system in use can be seen with the cytokine profile secreted by immune cells throughout the body. Although this is critical in shaping the immune response, researchers have little control over what cytokines are produced as it depends on the disease, setting, and receptor type. SynNotch has been used to remedy this issue by producing certain cytokines that initiate the proper response against a certain foreign body. For example, researchers installed a CD19 sensing synNotch receptor with the transcriptional element for one single stimulatory cytokine: IL-2. Sure enough, this was the only cytokine produced when the antigen CD19 was present. This was also shown to work with several other cytokines including the immunosuppressive cytokine IL-12. Another example includes using synNotch to locally deliver therapeutic antibodies so that they can be produced close to the tumor, potentially increasing their effectiveness. These antibodies generally target inhibitory receptors on other T-Cells that cause them to not be able to effectively target tumors and they actually enhance the activity of tumor-infiltrating T-Cells in addition to the therapeutic ones. To put this into use, researchers used a GFP synNotch receptor to successfully generate the inhibitory receptors like Pembrolizumab and CTLA-4 only when GFP was present. The T-Cells then showed the ability to mass produce the antibodies which bound themselves to their intended targets. Other specific payloads that synNotch has been used to deliver are apoptosis-inducing ligands, adjuvants (microbial products that stimulate the immune response), and even immunosuppressive agents if need be.

SynNotch receptors can also control cell differentiation in various T-Cells so that they develop a phenotype that is helpful in the context in which the cell is present. In initial tests, researchers used this system to transform fibroblasts (not T-Cells, but common cells that provide structure to cell tissue) into myoblasts (cells that have 
differentiated into muscle cells through myogenesis and later become a part of the skeletal muscle). They created fibroblasts with an anti-CD19 synNotch that had MyoD- the transcription factor that leads to myogenesis and they engineered sender fibroblasts cells that contained the CD19 ligand. After inserting the sender fibroblasts with control cells, the researchers found that only the fibroblasts that had come in contact with the sender fibroblasts that had CD19 ended up expressing the MyoD (showing how synNotch has the ability to regulate cell fate). This system has shown the potential to be compatible in a similar manner with T-Cells. Two helper CD4+T-Cell fates are T helper cell 1 (Th1) and T helper cell 2 (Th2), respectively controlled by transcription factors Tbet and GATA3. The need for skewing differentiation towards the IFN (cytokine mediators) producing Th1 cells is important for cancer clearance as these T-Cells activate other innate immune cells that help in fighting cancers. As a result, researchers tried to use a synNotch to provide antigen-dependent control of T-Cell differentiation by controlling the levels of Tbet in response the tumor antigen CD19 (Figure 2B). After testing, they noticed a skewed distribution of over 60\% cells having the Th1 phenotype as opposed to Th2. This demonstrated the success of synNotch to drive antigen-dependent T-Cell differentiation. All of these abilities display how the synNotch receptor allows the immune system to transcend the limits of its natural purpose of fighting cancers and other pathogens.

Multiple synNotch receptors can also be installed into a single cell where both would need to be activated before a response occurs. Each would control one half of a transcription factor, similar to the "AND" gates discussed in the next section (Morsut et al., 2016). Two synNotch receptors can also be used in series; this is to induce a cell-tocell cascade of activation. For example, researchers used an anti-GFP synNotch which, when stimulated by the GFP ligand, would release the CD19 ligand. This would then activate an anti-CD19 synNotch receptor which would in turn induce a reporter protein called tagBFP. This allows for immune cells to be activated in layers, or in a spatially controlled manner. This gives synNotch receptors the unique ability to activate local responses within certain areas in multilayered body tissue.

Advancements similar to those made in CAR's can also be adapted into synNotch receptors to mitigate some of the equally familiar problems that CAR's experience. An example of this is installing a newly discovered intracellular sequence (QHGQLWF, named as RAM7, that is found in native Notch receptors) into synNotch T-Cells to significantly reduce ligand-independent activation (Yang et al., 2020). These types of changes enhance the safety of synNotch receptors even further. The findings made with these novel cell receptors have made a great difference in how patients with cancers are treated and have a considerable amount of potential that is yet to be tapped into.

\section{Antigen Recognition/Cell Activation}

One of the most important facets of immune cells is their ability to activate at the proper time and correctly recognize the cells that they are intended to attack. As stated earlier, many cancers lack a targetable antigen that is unique to them, posing the danger of T-Cells attacking regular body tissue, or bystander cells (Roybal et al., 2016). To attack these types of cancers, researchers have developed "synNotch CAR's" that can recognize combinations of antigens. In this system a synNotch receptor recognizes an antigen, allows the CAR to express itself, and then the CAR recognizes the antigen. This creates a two-antigen system ("AND" Gate) where both necessarily have to be present for the T-Cell to activate- the system will not activate when only one antigen is present (Williams et al., 2020) (Figure 3A). This novel system has an additional level of security and is useful because it forgoes the previous one-dimensional system that had one receptor targeting one antigen. An example of this can be seen when researchers used a GFP synNotch receptor to drive expression of a CD19 CAR (shown to very effective in clearing out tumor) These T-Cells were then exposed to K562 target cells with either only GFP, only CD19, or both. Predictably, the CAR was only expressed when GFP was present and activation of the entire cell only occurred when both antigens were present. 
Another specific example of this system in use is with the tumor mesothelioma, a type of cancer that develops in the tissue lining of the lungs and other organs. The antigen ALPPL2 functions as the initial target that can then be used in combination with MCAM or mesothelin which are the tumor specific targets (Hyrenius-Wittsten et al., 2021). The system was tested in mice that had been implanted with tumor cells. After testing ALPPL2 CAR T-Cells, MCAM CAR T-Cells, or ALPPL2 synNotch $\rightarrow$ MCAM-CAR circuit T-Cells, the researchers found the latter to have the most complete response in terms of eradicating the tumor cells. Also, since the CAR is not expressed until the synNotch

A

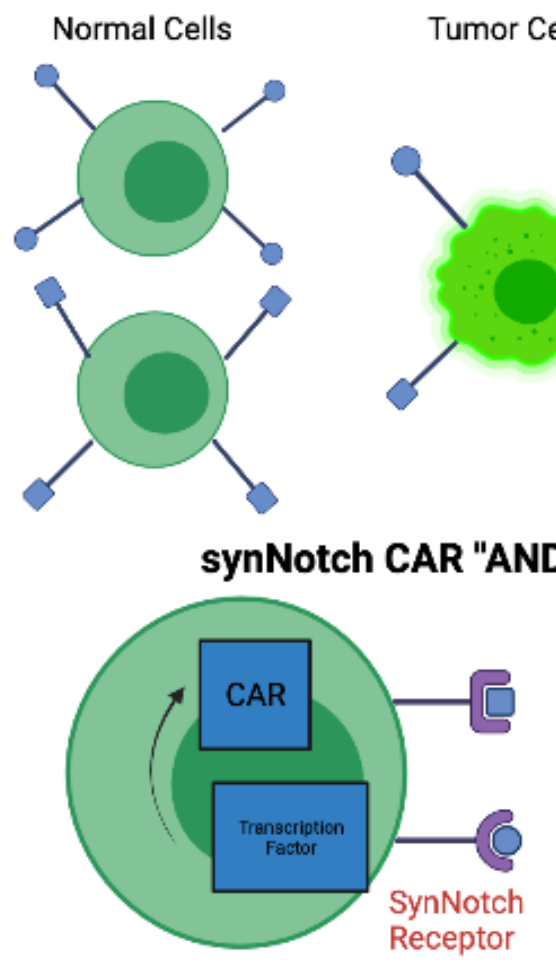

B 
Intracellular receptors are found within the cytoplasm of a cell and can recognize antigens only that cross the cell membrane. Unfortunately, CAR T-Cells can only sense extracellular antigens and this limits the sets of proteins that the cell can express as $75 \%$ of the proteome, the entire set of proteins that a cell can express at a given time, is found within the cell. Researchers have now created various internal-external cell recognition circuits in order to treat various diseases, including melanoma (a type of skin cancer). The intracellular antigen for this disease, MART1, is sensed by synthetic Notch receptors which allow it to be presented on the outside using major histocompatibility complex molecules (MHC's bind antigens from pathogens and display them on the cell surface for recognition by the T-Cell). T-Cells can be activated by this antigen but, like explained previously, this also leads to them attacking normal cells (specifically melanocytes - melanin producing cells found in the epidermis of the skin). To solve this problem, researchers found an extracellular surface antigen named MET that is highly expressed in melanoma cells but not normal melanocytes. They ended up creating a recognition circuit using synthetic notch receptors and normal T-cell receptors that required MART1 and MET antigens to be present so that T-cells would only activate to attack cancer cells. As demonstrated previously with the EGFRvIII, the MART1 is used to specify the range of cells that the T-cells attack and then the MET antigen is used to further specify and ensure that the only cell being attacked are the dangerous melanoma cells. The researchers found T-cells with these circuits to be more precise in terms of what they attack and also found them to maintain the same effectiveness in attacking the melanoma cancer cells. Synthetic notch receptors that target intracellular antigens have come to be known as "in-notch" receptors and have been shown to work with TCR's (normal T-Cell receptors) and CAR's in other "AND gate" situations to only target "dual positive" cells.

Other types of combinatorial circuits used with this popular construct include "NOT" gates (antigens are sensed to tell the T-Cell not to kill that particular cell), “AND” gates with 3+ antigens, "OR" gates (T-Cells begin to kill when they come into contact with either of two different antigens), and more (including combinations of the ones listed above) (Figure 3B). This system helps to ensure the safety of engineered T-Cells and is crucial to its practicability. Researchers have used another type of synthetic notch receptors: OFF-Notch. These circuits initiate T-Cell death (apoptosis) when initiated and are useful to block the rapid downstream T-Cell proliferation that occurs when they are activated. This OFF-Notch system was used in coordination with another "AND gate" system of two synthetic notch receptors. The "AND gate" would be used to facilitate T-Cell activation while the OFF-Notch would be used to prevent activation. The OFF-Notch receptor searched for the antigen HER 2 while the two synthetic notch receptors searched for the antigens GFP and CD19 respectively. Researchers tested this system with K562 tumor cells and found that when HER 2 was present the tumor cells were spared and T-Cells were killed while when GFP and CD19 were present target tumor cells were killed. This shows that the researchers were able to create a negative recognition circuit that prevents various CAR activity.

The “AND” gate system with synNotch CAR's can also be utilized with 3+ antigens. This system was tested in mice using a dual tumor model where one group of K562 tumor cells had 2 antigens (MET+/HER2+) and were the control while another group of tumor cells had 3 antigens (MET+/EGFR+/ HER2+) and were the target. The reasearchers found that the control tumor cells were ignored while the T-Cells effectively killed the target tumor cells, showing the effectiveness of the 3+ antigen "AND gate". Another example of one of these gates is the "OR" gate where TCells begin to kill when they come into contact with either of two different antigens. This can then also be combined with "AND gates" to create even more complex functions (this would be where one antigen is necessary for activation and it would need to be combined with an option of two others).

\section{Other Methods to Improve Recognition}

There are several other changes that have been made to T-Cells in order to modify the ways in which they activate and recognize their targets. For example, in order to make synNotch CAR T-Cells even safer, researchers have developed T-Cells that use synNotch CAR's to sense the density of antigens (Hernandez Lopez et al., 2021) (Figure 4A). For example, HER2 and EGFR are examples of antigens that are widely expressed on tumors but cannot be targeted because of their presence, albeit lower expression, on normal cells. This scenario resulted in researchers wanting to 
program T-Cells to have a sigmoidal (or "all or none") response where they only activate when there are a lot of antigens present. In this circuit, the synNotch is low-affinity which means it only expresses the CAR when it senses a lot of its antigen. Then, the CAR is high-affinity which means it targets anything with even a few of the antigens (this second step being an additional level of security). Here, the high-affinity synNotch acts as a filter that creates the "ultrasensitive threshold" which is useful and gives researchers a whole new slew of antigens that they can target.

Similar to this is the idea of using soluble ligands (dissolved in the bloodstream and different from normal surface antigens) to activate CAR T-Cells, again, in order to give researchers more control over the engineered cells (Chang et al., 2018) Based on this idea, researchers now can stimulate T-Cells with more control and freedom. As an example, they used the CD19 ectodomain from other HEK293 T-Cells to stimulate CD19 CAR cells to produce cytokines. This ectodomain is the part of a membrane protein that usually interacts with surfaces/leads to signal transduc-

A

Antigen Density Recognition

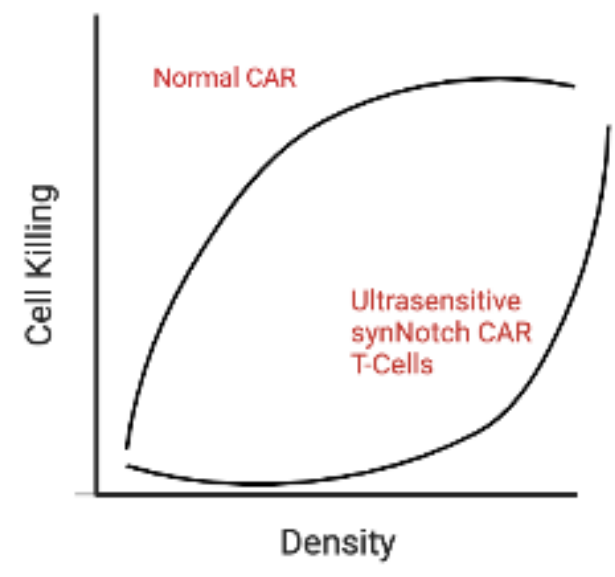

B Delivering CAR Targets to a Tumor via Viral Delivery

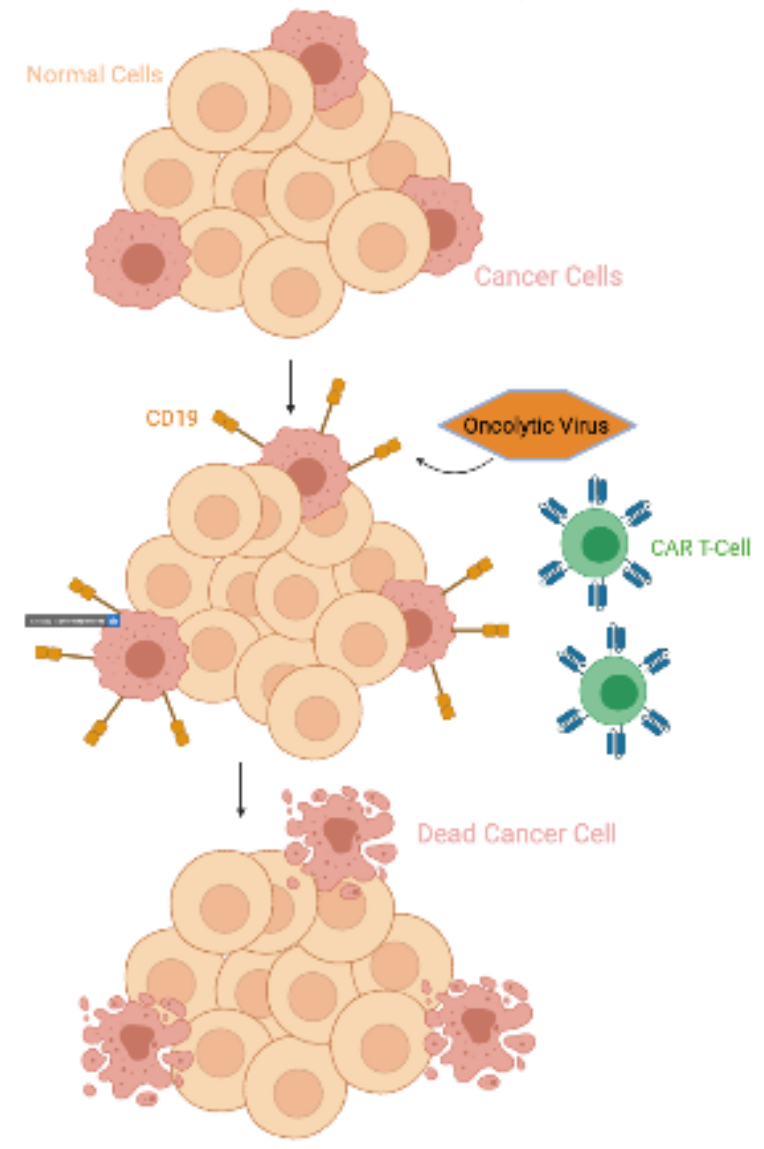

Figure 4: (A) This figure demonstrates how the cell killing of a T Cell with an ultrasensitive threshold is controlled. As opposed to a gradual increase, this system attacks at high levels only when a large density of antigens is present (B) Display of the process by which an oncolytic virus can be used to selectively deliver antigens to tumor cells. This allows them to be targeted by anti-CD19 CAR T-Cells and become eradicated. Created using BioRender.

tion and it proved to be effective in stimulating the CAR T-Cells.

Small molecules that usually have more of a pharmaceutical function can also be used to control the activity of engineered cells (Wu et al, 2015). Rapalog (modified version of rapamycin, an anti-fungal antibiotic) has been used in combination with a CD19 antigen in an "ON-switch" CAR. Both the antigen and the rapalog must be present to 
stimulate the action which could include cytokine production, cell proliferation, or even tumor killing. For tumor killing, the cells were first tested in a laboratory. Over the first 22 hours, the CD19 ON-switch CAR showed no activity in the presence of CD19. Killing of the target cells only ensued when $500 \mathrm{nM}$ of rapalog was added; in fact, the degree of killing could even be titrated/controlled by the amount of rapalog added and it could be stopped after a reset period if there was no rapalog present. This model was also tested with mice containing K562 tumor cells and proved to be reliable. This leads to the possibility of T-Cells possibly using more simple and reliable methods to target cells in the future instead of antigens. An example could be possibly only being activated by certain drugs - this would give researchers complete control over the T-Cells, if ever a need for this arises. However, the issue with this development is the lack of practically usable controller drugs like rapalog as they don't have the proper pharmacokinetic properties, but further advancements may make this system more prominent in the future. Other tools may also be considered, as seen in a recent example of researchers using pulses of light to stimulate CAR T-Cells (with high levels of cytotoxicity against cancer cells) using a new program known as LINTAD - light inducible nuclear translocation and dimerization system for gene regulation (Huang et al., 2020).

Another approach to activating T-Cells is using an oncolytic virus to selectively deliver surface antigens to cancers (Aalipour et al., 2020) (Figure 4B). This strategy is unique because in addition to the synthetically designed receptor being used, the cancer cells are also being modified. The researchers used an oncolytic virus to deliver antigens to the cancer for easier targeting. These viruses can infect and even replicate within cancer cells, helping to reprogram the immunosuppressive microenvironment (serious issue that was mentioned earlier), while being FDA approved. The combination of the CD19-containing virus and CD19 CAR T-Cells showed strong levels of toxicity in experiments and were able to eliminate target cells within 48 hours. This is another strategy that grants researchers high levels of control over the synthetically designed receptors while making it easier for them to activate at the proper time(s). In all, there are multiple ways to recognition systems of T-Cells in order and these will be useful in ensuring the

\section{CAR/ADR T-Cells} them.

\section{Extended Uses}

design the activation and to improve their function as a whole protection of every patient who uses

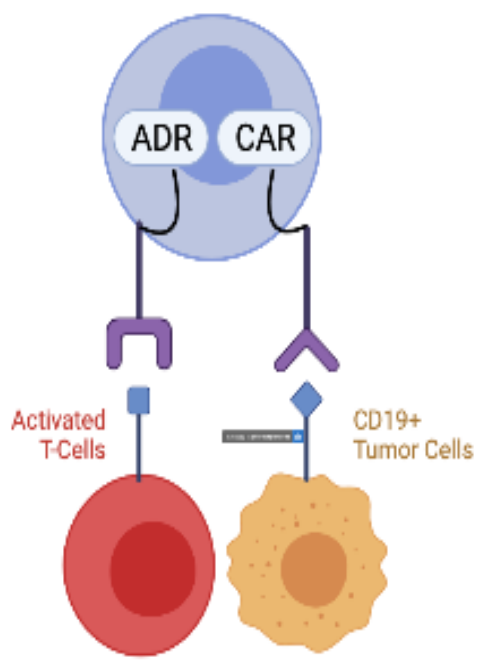

Figure 5: The CAR/ADR T-Cells have a dual-targeting system that allows them to resist host immune rejection. The CAR attacks cancer cells as normal, but the ADR receptor also allows for the T-Cell to resist rejection by eliminating attacking lymphocytes. Created using BioRender. 
One key aspect of the synthetic receptors described above is the adaptability and versatility of the functions thatthey perform. Normal T-Cells can also be modified in different ways. For example, TRuCs (T-Cell receptor Fusion Constructs), or T-Cell Receptors (TCR) combined with an antibody-based binding domain, have shown to be as effective as CAR T-Cells while releasing less cytokines and being better at eliminating liquid tumors (Baeurle et al., 2019). These changes also became integral to the body's endogenous TCRs as opposed to CAR's which are standalone receptors; this is because they essentially reprogram TCR's to target surface antigens. In a mouse model, these receptors showed more potent anti-tumor activity as compared to CAR's and even have more capabilities in the field of cell signaling. This helps to show that with more research and development, beneficial changes other than CAR's and synNotch receptors can be made to the natural immune system.

Researchers are also developing new and more innovative ways to mitigate the issues discussed earlier about CAR T-Cells (like off-target toxicity). New "gene circuits" have been installed in T-Cells that allow for a one-time switch in the state of CAR T-Cells via the use of an FDA approved drug; the effects even last after the drug has been removed (Chakravarti et al, 2019). A newly created gene switch, called a "FLEx switch" is responsible for the basis of this technique. It was created from work done with recombinases, enzymes known to be useful in controlling gene systems in cells. These gene circuits are most important for making permanent changes to T-Cells, such as shutting down a particular gene or turning it on. They also help to reduce the drug exposure for a patient while also not fully killing the T-Cells like other tools, including kill switches.

The manufacturing of synthetically modified T-Cells is an extremely precarious process. In fact, when many of these cells enter the body in order to help its immune response, other active T-Cells and NK Cells reject the cell (Mo et al, 2020). This often necessitates modified cells to be patient specific and this limits the full capability of synthetic immunology by making processes expensive and difficult to implement. As a result, researchers have developed an alloimmune defense receptor (ADR) that can sense 4-1BB, a surface antigen that active lymphocytes display for a short period. By equipping modified T-Cells that enter the body with this receptor, researchers are able to make sure that these cells are accepted by the immune system (Figure 5). This is because they can now defend themselves against active lymphocytes trying to destroy them while sparing resting ones. This model has shown to work in mice models, where researchers developed cells containing CAR's and an ADR to eliminate solid cancers. The ADR has huge promise as it provides hope to the potential of there being one common bank of modified T-Cells for all to use with ease of access and availability.

Finally, researchers have also begun to actually modify the receptors and other features of T-Cells in a more effective and time-saving manner. To create newly modified T-Cells, researchers have created a multitude of different "gene constructs". These need to be tested in vivo (inside an organism) to see which are the best for mass use (Roth et al, 2020). Unfortunately, in the past researchers have had trouble finding very effective means of testing such constructs to compare/contrast their impact in cells. However, recently researchers have used "pooled knock in screens" to test gene constructs that were best at enhancing cell fitness in an experimental setting. As implied, this technique allows them to test several different DNA sequences at one time to see which would be best at increasing the fitness of T-Cells. This powerful new medium uses CRISPR targeting (a type of genetic engineering technique that edits the genome of organisms) to introduce a 36-member library of constructs into a particular location in the human genome to compare T-Cells with various templates against each other. These templates include novel types of receptors, intracellular domains, metabolic regulators, and more. A specific example of an effective construct was one that led to the development of a TGF-bR2-41BB CAR that enhanced cell fitness in cells facing suppressive cytokines in vitro (in a laboratory) and reduced tumor accumulation while expressing key cytokines in vivo with mice. In all, these developments show that engineering different aspects of the immune system to be more productive is something that will be continued for years to come as there is no limit to the elaborate manners in which we can improve our body's defensive systems. 


\section{Other Types of Immune Cells}

Although the majority of work done in synthetic immunology has been done with lymphocytes and T-Cells in particular, other types of immune cells can be modified as well. For example, dendritic cells (antigen presenting cells) are known to be used in vaccines to combat tumor growth. Researchers have found type 1 DCs (cDC1's, where DC stands for dendritic cells) as the best subset of DC's to use in vaccines to promote anti-tumor immunity. Therefore, they have tried to revise the immunobiology of these cells to make them even more effective (Calmeiro et al, 2020). The cDC1 cells usually help induce lymphocytes to attack tumors so strategies aimed at getting them mobilized to the tumor microenvironment and allowing them to activate and expand are crucial in making them more effective in anti-tumor immunotherapy. However, in the long term, more work still needs to be done in this field to understand the impacts of DC's in vaccines (such as how they help attack the tumor and how the tumor impacts them).

Another interesting aspect of editing performed with other types of immune cells is using neutrophils and their ability to perform phagocytosis (digesting other harmful particles to destroy them) to potentially carry encapsulated substances with medicinal properties (Nikitina et al, 2020). Currently, tests have shown the neutrophils to initially carry the capsules in accordance with their size while maintaining visibility and the ability to move. However, long term these abilities decreased and cells were even destroyed. This shows the potential of the system but the necessity of further research to be performed.

Macrophages are another group of key players in our immune systems - they are versatile cells that have many phenotypes and are found in all types of tissue (Poltavets et al, 2020). For example, they have a pro-inflammatory state known as M1 (prompt the processes of inflammation and cell death) and an anti-inflammatory state known as M2 (prompt the processes of regeneration, angiogenesis, and even extracellular matrix remodeling) that they can modify based on the tumor microenvironment. This has opened the door for researchers to try and control this shifting on their own. In all, the omnipresence of macrophages makes them the perfect target for use in cell therapies but more study will be needed for practical use to occur. Although T-Cells may be the current focus of synthetic immunology, the untapped potential that other immune cells bring to the table make them impossible to ignore for study in the future.

\section{Implications}

The majority of information presented in this review reflects the fundamental aspects of synthetic immunology. Techniques described towards the latter end of the text reflect more novel uses/advancements that researchers have engineered to be used in synthetic cells. The paper strives to present the current research in the field while showcasing the wide range of prospective constructs/uses that could play a major part in future treatments.

The immune system is an extremely complex network that requires many cell types to work together to eradicate an invader/cancer. The field of synthetic immunology is an astounding field as it leverages small changes to a single cell type and shows that the immune system can be altered to attack a new antigen. Additionally, the research discussed denotes the high level of flexibility that the immune system offers; researchers have the ability to leverage simple modifications to make a variety of complex treatments. In the future, existing systems may be further modified in many ways to protect patients with more reliable types of therapy, highlighting the seemingly limitless potentials of the field.

\section{Conclusion}

Cancers and other foreign invaders to the human body pose life-threatening danger to all people around the globe. The immune system, despite its multilayered attempts to protect us, can get shut down very easily and essentially become ineffective in protecting the body against severe tumor growth. The field of synthetic immunology is vital in order for 
researchers to discover monumental synthetic constructs that could potentially enhance the immune system and aid it in its goal of keeping the body healthy. Key receptor enhancements such as CAR's/synNotch receptors and methods to specify when T-Cells activate (plus who they attack) can be combined with a wide variety of other changes to help bolster the effectiveness of the immune system. These recent developments have come a long way in helping to combat the spread of cancers and other pathogens. Overall, more complex studies relating to synthetic immunology will enable it to further transform the immune system as we know it today.

\section{Future Work}

One particular area in which more development is extremely important is determining and exploring the detrimental effects that may come with the manipulation of these cells. Potentially, advanced receptors in the future would be able to perform more complex functions while giving researchers user control with the touch of a button (developing a system allowing researchers to turn all modified cells on/off). It would also be interesting to see more work done in enhancing the way receptors sense the target cells that they plan to attack. Perhaps modified immune cells could recognize something different about the tumor (other than an antigen), such as its chemical composition or simply the activity of their abnormal growth.

\section{Limitations}

Given the safety risk and reduced efficacy for solid tumors, a lot of this research has not yet been implemented for the treatment of human patients. As a result, there have not been many case studies in vivo as to how the modifications described could have an impact on human health. However, many companies are now investing in technologies like the ones described here to improve the efficacy and safety of the engineered cells such that they can be tested in humans in the near future.

\section{Acknowledgements}

I would like to thank the Polygence platform for providing me with the opportunity to work on this project. Additionally, I would like to thank my mentor Pavithran Ravindran who was a great guide through the process of reading papers and being able to write my own.

\section{References}

Alipour et al., A. (n.d.). Viral Delivery of CAR Targets to Solid Tumors Enables Effective Cell Therapy. Molecular Therapy: Oncolytics, 17. https://doi.org/10.1016/j.omto.2020.03.018.

Baeuerle, P. A., Ding, J., Patel, E., Thorausch, N., Horton, H., Gierut, J., ... Hofmeister, R. (2019). Synthetic TRuC receptors engaging the complete $\mathrm{T}$ cell receptor for potent anti-tumor response. Nature Communications, 10(1). https://doi.org/10.1038/s41467-019-10097-0

Calmeiro, J., Carrascal, M. A., Tavares, A. R., Ferreira, D. A., Gomes, C., Falcão, A., ... Neves, B. M. (2020). Dendritic Cell Vaccines for Cancer Immunotherapy: The Role of Human Conventional Type 1 Dendritic Cells. Pharmaceutics, 12(2), 158. doi:10.3390/pharmaceutics12020158

Chang, Z., Lorenzini, M., Chen, X. et al. Rewiring T-cell responses to soluble factors with chimeric antigen receptors. Nat Chem Biol 14, 317-324 (2018). https://doi.org/10.1038/nchembio.2565 
Chakravarti, D., Caraballo, L. D., Weinberg, B. H., \& Wong, W. W. (2019). Inducible Gene Switches with Memory in Human T Cells for Cellular Immunotherapy. ACS Synthetic Biology, 8(8), 1744-1754. https://doi.org/10.1021/ acssynbio. $8 \mathrm{~b} 00512$

Cho, J. H., Collins, J. J., \& Wong, W. W. (2018). Universal Chimeric Antigen Receptors for Multiplexed and Logical Control of T Cell Responses. Cell, 173(6), 1426-1438.e11. https://doi.org/10.1016/j.cell.2018.03.038

Choe, J. H., Watchmaker, P. B., Simic, M. S., Gilbert, R. D., Li, A. W., Krasnow, N. A., Downey, K. M., Yu, W., Carrera, D. A., Celli, A., Cho, J., Briones, J. D., Duecker, J. M., Goretsky, Y. E., Dannenfelser, R., Cardarelli, L., Troyanskaya, O., Sidhu, S. S., Roybal, K. T., Okada, H., ... Lim, W. A. (2021). SynNotch-CAR T cells overcome challenges of specificity, heterogeneity, and persistence in treating glioblastoma. Science translational medicine, 13(591), eabe7378. https://doi.org/10.1126/scitranslmed.abe7378

Choe, J. H., Williams, J. Z., \& Lim, W. A. (2020). Engineering T Cells to Treat Cancer: The Convergence of Immuno-Oncology and Synthetic Biology. Annual Review of Cancer Biology, 4(1), 121-139. https://doi.org/10.1146/annurev-cancerbio-030419-033657

Hernandez-Lopez, R. A., Yu, W., Cabral, K., Creasey, O., del Pilar Lopez Pazmino, M., Tonai, Y., De Guzman, A., Mäkelä, A., Saksela, K., Gartner, Z. J., \& Lim, W. A. (2021). T cell circuits that sense antigen density with an ultrasensitive threshold. BioRxiv, 2021.01.21.427654. https://doi.org/10.1101/2021.01.21.427654

Herzig, E., Kim, K. C., Packard, T. A., Vardi, N., Schwarzer, R., Gramatica, A., Deeks, S. G., Williams, S. R., Landgraf, K., Killeen, N., Martin, D. W., Weinberger, L. S., \& Greene, W. C. (2019). Attacking Latent HIV with convertibleCAR-T Cells, a Highly Adaptable Killing Platform. Cell, 179(4), 880-894.e10. https://doi.org/10.1016 /j.cell.2019.10.002

Hyrenius-Wittsten, A., Su, Y., Park, M., Garcia, J. M., Alavi, J., Perry, N., Montgomery, G., Liu, B., \& Roybal, K. T. (2021). SynNotch CAR circuits enhance solid tumor recognition and promote persistent antitumor activity in mouse models. Science translational medicine, 13(591), eabd8836. https://doi.org/10.1126/scitranslmed.abd8836

InformedHealth.org [Internet]. Cologne, Germany: Institute for Quality and Efficiency in Health Care (IQWiG); 2006-. What are the organs of the immune system? [Updated 2020 Jul 30]. Available from:

https://www.ncbi.nlm.nih.g ov/books/NBK279395/

Kuwana, Y., Asakura, Y., Utsunomiya, N., Nakanishi, M., Arata, Y., Itoh, S., Nagase, F., \& Kurosawa, Y. (1987). Expression of chimeric receptor composed of immunoglobulin-derived $\mathrm{V}$ regions and T-cell receptor-derived $\mathrm{C}$ regions. Biochemical and biophysical research communications, 149(3), 960-968. https://doi.org/10.1016/0006$\underline{291 \times(87) 90502-x}$

Mo, F., Watanabe, N., McKenna, M.K. et al. Engineered off-the-shelf therapeutic T cells resist host immune rejection. Nat Biotechnol 39, 56-63 (2021). https://doi.org/10.1038/s41587-020-0601-5

Morsut, L., Roybal, K. T., Xiong, X., Gordley, R. M., Coyle, S. M., Thomson, M., \& Lim, W. A. (2016). Engineering Customized Cell Sensing and Response Behaviors Using Synthetic Notch Receptors. Cell, 164(4), 780-791. https://doi.org/10.1016/j.cell.2016.01.012 
Nikitina, M.Y., Ponomareva, A.A., Daminova, A.G. et al. Viability, Ultrastructure, and Migration Activity of Neutrophils after Phagocytosis of Synthetic Microcapsules. Cell Tiss. Biol. 14, 275-285 (2020).

https://doi.org/10.1134/S1990519X20040069

Poltavets, A. S., Vishnyakova, P. A., Elchaninov, A. V., Sukhikh, G. T., \& Fatkhudinov, T. K. (2020). Macrophage Modification Strategies for Efficient Cell Therapy. Cells, 9(6), 1535. doi:10.3390/cells9061535

Roth et al., Pooled Knockin Targeting for Genome Engineering of Cellular Immunotherapies, Cell (2020), https://doi.org/10.1016/j.cell.2020.03.039

Roybal, K. T., \& Lim, W. A. (2017). Synthetic Immunology: Hacking Immune Cells to Expand Their Therapeutic Capabilities. Annual review of immunology, 35, 229-253. https://doi.org/10.1146/annurev-immunol-051116052302

Roybal, K. T., Rupp, L. J., Morsut, L., Walker, W. J., McNally, K. A., Park, J. S., \& Lim, W. A. (2016). Precision Tumor Recognition by T Cells With Combinatorial Antigen-Sensing Circuits. Cell, 164(4), 770-779. https://doi.org/10.1016/j.cell.2016.01.011

Roybal, K. T., Puchner, E. M., Onuffer, J., \& Lim, W. A. (2015). Remote control of therapeutic T cells through a small molecule-gated chimeric receptor. Science (New York, N.Y.), 350(6258), aab4077.

https://doi.org/10.1126/science.aab4077

Roybal, K. T., Williams, J. Z., Morsut, L., Rupp, L. J., Kolinko, I., Choe, J. H., Walker, W. J., McNally, K. A., \& Lim, W. A. (2016). Engineering T Cells with Customized Therapeutic Response Programs Using Synthetic Notch Receptors. Cell, 167(2), 419-432.e16. https://doi.org/10.1016/j.cell.2016.09.011

Rupp, L.J., Schumann, K., Roybal, K.T. et al. CRISPR/Cas9-mediated PD-1 disruption enhances anti-tumor efficacy of human chimeric antigen receptor T cells. Sci Rep 7, 737 (2017). https://doi.org/10.1038/s41598-017-00462-8

$\underline{\mathrm{Wu}, \mathrm{C}}$.

Williams, J. Z., Allen, G. M., Shah, D., Sterin, I. S., Kim, K. H., Garcia, V. P., Shavey, G. E., Yu, W., Puig-Saus, C., Tsoi, J., Ribas, A., Roybal, K. T., \& Lim, W. A. (2020). Precise T cell recognition programs designed by transcriptionally linking multiple receptors. Science (New York, N.Y.), 370(6520), 1099-1104.

https://doi.org/10.1126/science.abc6270

Wu, C. Y., Roybal, K. T., Puchner, E. M., Onuffer, J., \& Lim, W. A. (2015). Remote control of therapeutic T cells through a small molecule-gated chimeric receptor. Science (New York, N.Y.), 350(6258), aab4077.

https://doi.org/10.1126/science.aab4077

Yamamoto, T. N., Lee, P. H., Vodnala, S. K., Gurusamy, D., Kishton, R. J., Yu, Z., Eidizadeh, A., Eil, R., Fioravanti, J., Gattinoni, L., Kochenderfer, J. N., Fry, T. J., Aksoy, B. A., Hammerbacher, J. E., Cruz, A. C., Siegel, R. M., Restifo, N. P., \& Klebanoff, C. A. (2019). T cells genetically engineered to overcome death signaling enhance adoptive cancer immunotherapy. The Journal of clinical investigation, 129(4), 1551-1565.

https://doi.org/10.1172/JCI121491 